\title{
Complete right bundle branch block of unique behavior: What is the mechanism?
}

\author{
Finn Akerström, Miguel A. Arias, Marta Pachón, Alberto Puchol \\ Cardiac Arrhythmia and Electrophysiology Unit, Department of Cardiology, \\ Hospital Virgen de la Salud, Toledo, Spain
}

\section{Case summary}

A 59-year-old hypertensive man presented to the emergency department with an episode of dizziness without syncope. A surface 12-lead electrocardiogram (ECG) showed sinus rhythm at $82 \mathrm{bpm}$ with a $2: 1$ complete right bundle branch block (RBBB) pattern (Fig. 1). The patient presented a full spontaneous recovery and was subsequently discharged and referred for ambulatory diagnostic work-up. Echocardiography presented moderate septal hypertrophy and no other structural abnormalities. Continuous 24-h 3-lead Holter monitoring was performed which revealed sinus rhythm with narrow QRS complexes at $42 \mathrm{bpm}$ (Fig. 2A), 2:1 RBBB at $60 \mathrm{bpm}$ (Fig. 2B) and 1:1 RBBB at $38 \mathrm{bpm}$ (Fig. 2C). Following this, an exercise stress test was carried out during which a 3:2 RBBB pattern was observed at the maximum heart rate of $116 \mathrm{bpm}$ (Fig. 3). What is the mechanism explaining the different patterns of transient RBBB observed and how can one relate this to the underlying sinus rate?

\section{Commentary}

Intermittent bundle branch block may be caused by several mechanisms namely tachycardiadependent (phase 3) block, acceleration-dependent block, bradycardia-dependent (phase 4) block and concealed conduction [1]. In our patient, the changes from normal right bundle branch (RBB) conduction at a heart rate of $42 \mathrm{bpm}$ (Fig. 2A) to 2:1 RBBB at a relatively faster heart rate $(60 \mathrm{bpm}$; Fig. $2 \mathrm{~B})$ is likely to be due to acceleration-dependent block. Normally, as the heart rate accelerates, the His-Purkinje system (HPS) effective refractory period

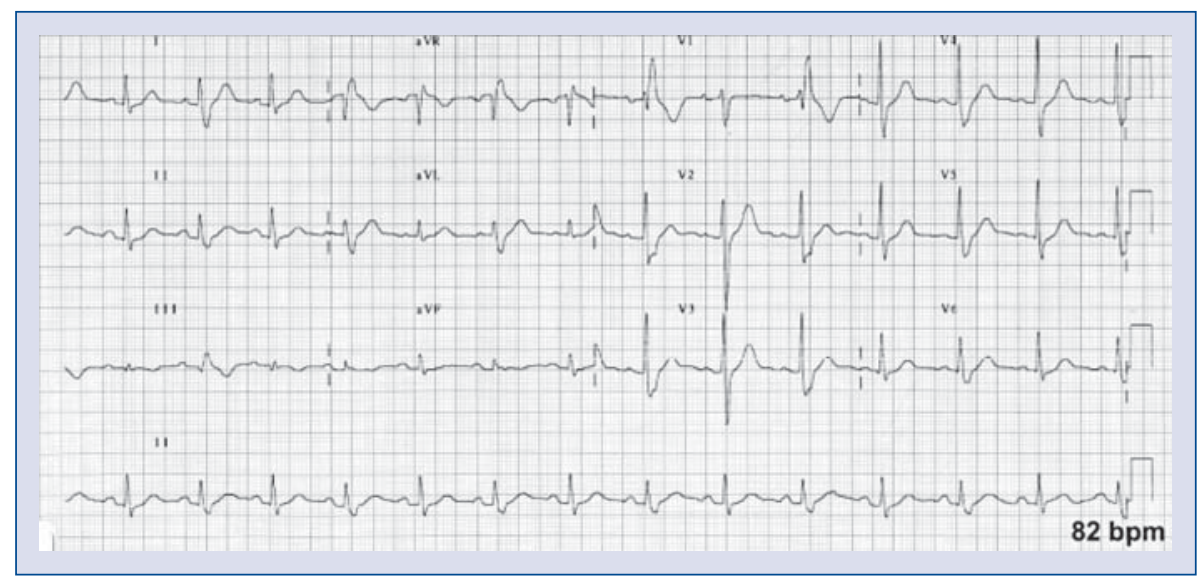

Figure 1. Baseline 12-lead ECG on admission showing a 2:1 right bundle branch block pattern. The sinus rate is $82 \mathrm{bpm}$.

Address for correspondence: Dr. Miguel A. Arias, Unidad de Arritmias y Electrofisiología Cardiaca, Avda. Barber 30, Planta Semisótano, 45004, Toledo, Spain, tel/fax: +34925265492, e-mail: maapalomares@secardiologia.es

Received: 11.05.2012

Accepted: 23.05.2012 


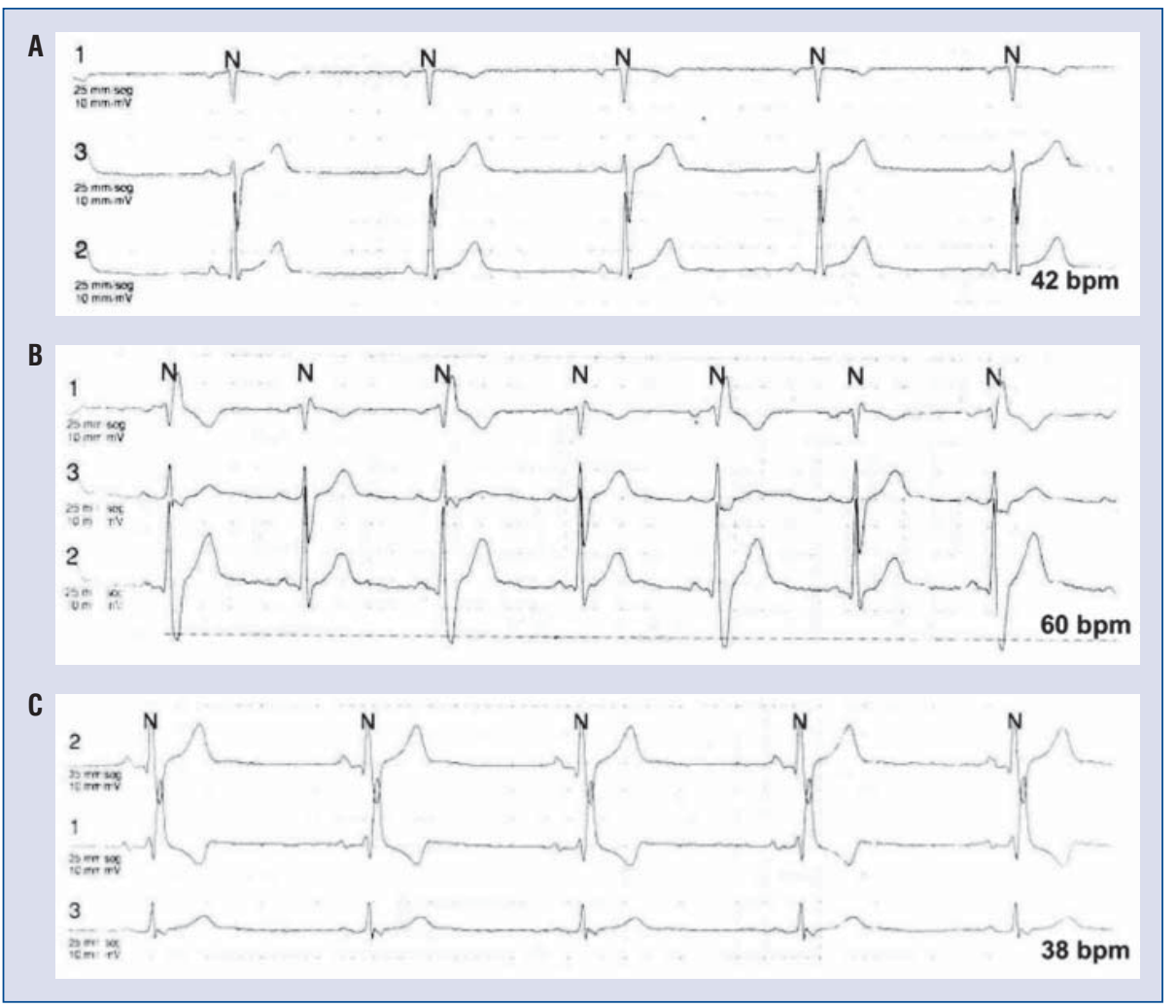

Figure 2. Continuous 24-h ambulatory 3-lead Holter monitoring revealing sinus rhythm with narrow QRS complexes at $42 \mathrm{bpm}$ (A), 2:1 right bundle branch block at $60 \mathrm{bpm}$ (B) and 1:1 right bundle branch block at $38 \mathrm{bpm}$ (C).

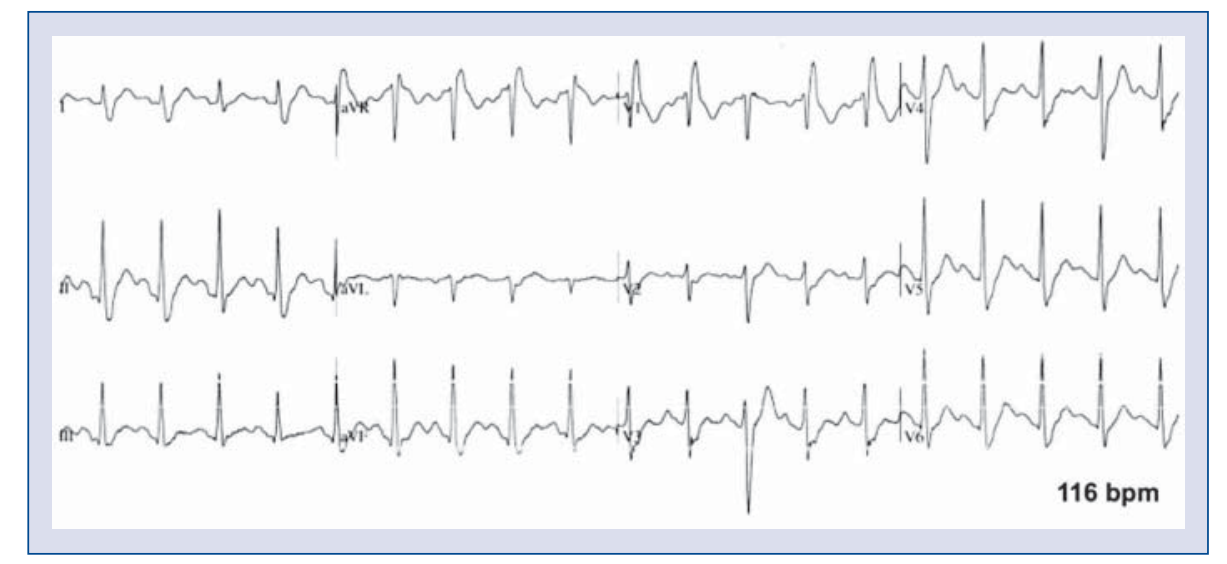

Figure 3. Twelve-lead ECG recording during exercise stress testing. A sinus tachycardia with a 3:2 right bundle branch block pattern is observed.

shortens thereby preserving normal conduction. Acceleration-dependent block occurs at critically increasing heart rates (but still below the action potential duration) when the action potential of the, often diseased, bundle branch fails to shorten in response to heart rate acceleration [1]. When this type of block occurs at relatively low heart rates, such as $60 \mathrm{bpm}$, which was the case in our patient, the HPS is clearly damaged.

But how can we explain the 2:1 (Fig. 2B) and the 3:2 RBBB (Fig. 3) patterns observed in our patient during the Holter recording? A probable mechanism would be an atypical Wenckebach-type second-degree block in the RBB [2]. Anterograde 
conduction through the RBB is progressively impaired due to some area of prolonged refractoriness. At a heart rate of $60 \mathrm{bpm}$ every second impulse fails to engage this area, allowing time for recovery for the following beat which conducts normally thereby producing a narrow QRS complex. The 3:2 RBBB pattern, occurring at a higher rate $(116 \mathrm{bpm})$ can be explained by the same mechanism with the difference that the first and second wide QRS complexes represents variable penetration and conduction block, respectively, followed by a normally conducted impulse with a narrow QRS complex.

Another plausible underlying mechanism for the intermittent RBBB observed might be tachycardia-dependent (phase 3) block. This phenomenon is caused by encroachment of the impulse on the relative refractory period (commonly during phase 3 of the action potential) of the preceding impulse. The block is perpetuated due to concealed transseptal conduction from the contralateral bundle, which renders the affected bundle refractory to the following beat [1]. The 2:1 and 3:2 RBBB patterns can be justified by bidirectional block, and alternating bidirectional and unidirectional block in the RBB, respectively. Nevertheless, this hypothesis is less likely since tachycardia-dependent (phase 3) block usually occur at much shorter cycle lengths than hereby reported.

The 1:1 RBBB observed when the heart rate decreased to $38 \mathrm{bpm}$ is suggestive of a bradycardia-dependent (phase 4) block as the underlying mechanism (Fig. 2C). This type of block is seen in diseased HPS during long cycles where gradual spontaneous depolarization during phase 4 takes place, resulting in the inactivation of sodium channels. As a result, the cell is then activated from a less negative membrane potential, causing impaired conduction. Bradycardia-dependent (phase 4) block usually affects the left bundle branch, possibly due to higher susceptibility to ischemic damage and a higher rate of spontaneous phase 4 depolarization of the left ventricular conduction system [1]. Nevertheless, this type of block has previously been reported to similarly occur in the RBB [3] and there is no reason why this fascicle could not also be affected as observed in our patient.
As a general consideration, since the different intermittent RBBB conduction blocks herein described were observed during a 24-h Holter recording and at an exercise stress test, the potential influence of the autonomic nervous system should be taken into account. Isoproterenol has been reported to both shorten the refractoriness of the affected bundle branch, thereby improving conduction related to tachycardia-dependent (phase 3) block, and to prolong the bradycardia-dependent (phase 4) block range [4]. The different patterns of intermittent RBBB (Fig. 2A-C) observed in the Holter recording all occurred during active hours, and therefore circadian rhythm related changes in catecholamine levels are less likely. Nevertheless, subtle changes in autonomic nervous tone might have contributed to the appearance of some of the intermittent RBBB observed e.g. promoting the transition from normal RBB conduction to a 1:1 RBB bradycardia-dependent (phase 3) block (Fig. 2C) despite a minor decrease in heart rate (42 to $38 \mathrm{bpm}$ ).

Finally, it might seem unlikely that the different types of conduction blocks that we have proposed all occurred in the same individual. However, each of them (acceleration-dependent block, progressive impairment of anterograde RBB conduction and bradycardia-dependent block) has been associated with diseased HPS and they are therefore likely to coexist when this type of cardiac abnormality is present.

Conflict of interest: none declared

\section{References}

1. Josephson ME. Intraventricular conduction disturbances. In: Clinical cardiac electrophysiology: Techniques and interpretations. $4^{\text {th }}$ Ed. Lippincott Williams and Wilkins, Philadelphia, PA, 2008: 110-139.

2. Gallagher JJ, Damato AN, Varghese PJ, Caracta AR, Josephson ME, Lau SH. Alternative mechanisms of apparent supernormal atrioventricular conduction. Am J Cardiol, 1973; 31: 362-371.

3. Arias MA, Domínguez-Pérez L, Pachón M, Rodríguez-Padial L. Second-degree 2:1 atrioventricular block with right bundle-branch block: What is the mechanism? Heart Rhythm, 2011; 8: 1650-1651.

4. Halpern MS, Chiale PA, Nau GJ et al. Effects of isoproterenol on abnormal intraventricular conduction. Circulation, 1980; 62: 1357-1364. 